\title{
Identifying regional variation in place visit behavior during a global pandemic
}

\author{
Grant McKenzie ${ }^{1}$ and Kevin M. Mwenda ${ }^{2,3}$ \\ ${ }^{1}$ Department of Geography, McGill University, Montréal, QC, Canada \\ ${ }^{2}$ Spatial Structures in the Social Sciences (S4), Brown University, Providence, RI, USA \\ ${ }^{3}$ Population Studies and Training Center, Brown University, Providence, RI, USA
}

Received: August 10, 2021; returned: September 17, 2021; revised: October 26, 2021; accepted: November 11, 2021.

\begin{abstract}
The emergence of the SARS-CoV-2 virus in 2019 lead to a global pandemic that altered the activity behavior of most people on our planet. While government regulations and public concern modified visitation patterns to places of interest, little research has examined the nuanced changes in the length of time someone spends at a place, nor the regional variability of these changes. In this work, we examine place visit duration in four major U.S. cities, identify which place types saw the largest and smallest changes, and quantify variation between cities. Furthermore, we identify socio-economic and demographic factors that contribute to changes in visit duration and demonstrate the varying influence of these factors by region. The results of our analysis indicate that the pandemic's impact on visiting behavior varies between cities, though there are commonalities found in certain types of places. Our findings suggest that places of interest within lower income communities experienced less change in visit duration than others. An increase in the percentage of younger, Black or Hispanic populations within a community also resulted in a smaller decrease in visit duration than in other communities. These findings offer insight into the factors that contribute to changes in visiting behavior and the resilience of communities to a global pandemic.
\end{abstract}

Keywords: visit duration, place type, time spent, COVID-19, pandemic, regional variability 


\section{Introduction}

The prevalence and variety of places of interest (POI) within a city tell us a lot about a city's inhabitants, visitors, design, and governance. The density of POI and mixture of place types (e.g., restaurants, parks, fire stations) within a region are valuable data, often used to inform urban planners and public policy makers as they devise or refine plans for a city. While a substantial body of literature has previously explored the spatial variety of place types and its influence on urban design $[65,13]$, another dimension of the city, namely time, has more recently experienced renewed interest. Much of this work has examined the times of day in which places of interest are in operation [54], or the popularity of places on typical days of the week or during major events [36]. In fact, Google introduced an exploratory feature into their Maps platform in late 2015 depicting the typical popularity of a place of interest by hour of the day and day of the week. While when and where people interact with places tells us a lot about the pulse of a city, an important interactive dimension has largely been ignored by the spatial data science research community, namely visit duration.

Knowing the visit duration, or the amount of time an individual spends at a place of interest, is informative for a variety of reasons. By knowing how long visitors typically spend at a certain type of place, one has a better idea of the relative importance of different place types. This knowledge can be used in the regional allocation of resources within a city, e.g., infrastructure spending, green space revitalization. If we observe an increase in the time people spend at a park, for example, additional funding can be allocated for public restrooms, garbage removal, safety officers, etc. Alternatively, municipal governments may elect to increase infrastructure spending to parks experiencing a decline in duration, with the goal of encouraging public engagement. Similarly, if a commercial coffee company discovers that customers, on average, spend less time at their downtown location than their uptown shop, they can further investigate the reasons and take action to incentivize a change in behavior.

Access to visit duration information is particularly useful when comparing place types between regions. Discovering that customers to family restaurants in New York City stay an average of 30 minutes longer than those in Los Angeles is quite insightful and opens up a new line of research related to explaining why these differences exist. What factors lead individuals and groups to behave differently towards the same place type in different cities? This is clearly of interest to marketing and industry professionals, as demonstrated through ongoing work in hospitality research [28, 27], but is also relevant to behavioral geographers and urban scientists. By investigating temporal duration we are able to further refine our understanding of activity behavior within a city. This complements much of the existing research on temporal popularity of places and the regional variability of mobility patterns [38].

The emergence of the novel coronavirus disease (COVID-19) in late 2019 caused significant disruption to the daily activity patterns of most individuals, as local and national governments enacted lock-downs, inter-regional travel was restricted, and businesses were shuttered. Within the spatial science communities, a body of research emerged focusing on identifying changes in human mobility patterns, case clustering, and outbreak event prediction. More recently we have seen a shift towards better understanding the social, economic, and health-related impacts of the pandemic on individuals in different geographic regions and from varied demographic backgrounds (see [12] for an overview). Still, little research has investigated the effect of the pandemic on visiting behavior at a high categor-

WWw.josis.org 
ical resolution, and across major metropolises. Which types of places were most impacted by the pandemic? Was a change in visit duration in one city also observed in another? Answering these questions takes a step towards better understanding a population's resilience and demonstrating that resilience is regionally variant.

In this work, we leverage access to large volumes of visit duration data in the United States just prior to the start of the pandemic as well as one year later, in the midst of the United States' third wave. These data offer an unprecedented opportunity to better understand changes in human activity due to the pandemic. Through analyses of these data, we are able to quantify change in visit activity for different place types in one dimension, and explore how these variations change between urban centers, in another. Through access to community socio-economic and demographic data, we identify nuanced relationships between certain population characteristics and a change in visit duration. This allows us to contribute to the growing body of literature related to the impact of pandemics on lowincome and under-represented populations in a unique way. With this in mind, the work presented in this paper will address the following three research questions.

RQ1 Prior to the COVID-19 pandemic, were there variations in place type visit durations between major U.S. cities? Our objective is to identify the place types whose visit durations are most and least regionally variant. To address this question we aggregate visit duration by place type for each of four major US cities. We report on the similarities between cities using a variety of statistical methods, highlighting the most similar and dissimilar place types.

RQ2 Are there quantifiable differences in place type visit durations before the pandemic (January 2020), and during the pandemic (January 2021)? Furthermore, does this difference vary regionally? We address this question by measuring the change in visit duration across place types and cities. Differences between and within cities are reported and unique examples are highlighted.

RQ3 What is the relationship between change in place type visit duration and the socio-economic and demographic characteristics of the communities in which POI are situated? To address this question we run a series of regression models to explore the interactions between socio-economic and demographic variables, place types, cities, and the change in visit duration. We report on the outcome of these analyses as well as the similarities and differences between place types and cities.

The remainder of this paper is organized as follows. Related works are presented in Section 2 followed by an overview of the datasets used in this analysis in Section 3. The methodology used in addressing the research questions is provided in Section 4 and the results are presented in Section 5. Finally, we discuss our analyses and results within a broader context in Section 6 and state our conclusions in Section 7.

\section{Related work}

Existing studies have explored various aspects of place-based visiting behavior. A lot of this work has been in the place recommendation domain with researchers developing novel methods to recommend a location based on previous places visited [43], neighborhood characteristics [32], and many others. Within the temporal dimension, much of the work 
has focused on temporal patterns as points in time without an explicit duration associated with a place visit. For instance, early work by Ye et al. [66] investigated the temporal visiting behavior of individuals to places of interest as reported through their locationbased social media check-ins, finding that temporal patterns varied considerably by POI tags. Research by McKenzie et al. [36] continued in this vein extracting unique temporal signatures for each place type and using these signatures to augment geolocating services. Both of these research directions represented temporal patterns as a single point in time, as virtually no geosocial media check-in platforms record visits as durations. This practice of using point-in-time-based temporal patterns has seen numerous contributions in recent years examining everything from residential vacancy rates [45] and criminal activity [47], to the temporal and environmental factors contributing to park visits [20].

A field of research that has considered visit duration for quite some time is Time Geography, specifically those contributions that develop or enhance space-time prisms. Spacetime prisms [41] are a conceptual model representing the spatiotemporal constraints of an individual, or group, as they go about their lives or accomplish specific tasks. At the heart of the space-time prism is the time that it takes to move between spaces (depending on mode, affordances, and speed of travel) as well as the visit duration or dwell time at specific locations. Researchers in this field have estimated dwell times at locations within a spacetime prism [55] as well as attempted to measure activity duration [26]. However, to the best of our knowledge, no research within this field has used the constraints of space-time prisms to differentiate dwell time or visit duration between different types or categories of places.

If we shift our focus explicitly to changes in visit duration, we find a number of studies have explored factors contributing to an increase or decrease in visit duration, with most targeting a single place type or industry. For example, work by De Freitas [8] identified atmospheric conditions that contributed to increased or decreased visit time at beaches. Research in the medical field has investigated the relationship between hospital and doctor's office visit duration with improved care [4] and patient satisfaction [46]. Research in the hospitality and management domains has extensively studied the relationship between restaurant visit duration, customer behavior [10], online reviews [23], and diner preferences [67]. Furthermore, visit duration to tourist attractions has been used in a number of cases as an attribute on which to build place-based and tourist recommendation systems [30,33]. On the other side of this topic, several computational projects have been developed to estimate visit duration at different place types using a variety of mobile sensor technologies [6,29], though the primary objectives of these projects are data collection and place type disambiguation.

Identifying similarities and differences between urban regions has been examined from a variety of perspectives. Early work by Uitenbroek et al. [58] identified differences between European cities based on the demographics and habitual behavior of individual inhabitants and groups. Other studies have explored the urban structure and morphology of the city as a basis for comparison to other cities [56, 25]. The combinations of place types in a city or neighborhood have proven to be useful in identifying regions [31], showing that spatiothematic context is an important dimension on which to compare cities. McKenzie et al. [37] explored the regional variation in temporal check-in behavior across cities in the United States demonstrating that there are important differences in when people visit place types depending on the city. Recent work by Tu et al. [57] has suggested that geosocial media check-ins combined with other user-contributed data can be used in the development 
of an urban vibrancy metric on which different urban regions can be compared. The work presented in this paper continues on this thread but focuses instead on the actual duration of visits and the impact of a global pandemic on visitation patterns.

The emergence of the COVID-19 pandemic has given rise to a large volume of research discussing the change in activity and mobility patterns attributed to the COVID-19 pandemic $[64,35]$. While much of this work has focused on developing platforms and tools for monitoring and predicting the course of the pandemic [2, 14], a growing body of literature is exposing the inequities in how the pandemic has impacted communities [3, 7, 22]. For instance, Benitez et al. [1] examined the relationship between confirmed COVID-19 infections, race, and ethnicity in six American cities including New York City and Chicago. The researchers found a higher percentage of both COVID-19 cases and related deaths in regions within American cities with higher percentages of Black and Hispanic residents, a finding corroborated by other researchers in this domain [21]. Recent work by Verma et al. [63] demonstrated that COVID-19 case count was significantly higher in lower-income neighborhoods within the United States. Similar findings have been reported in other parts of the world [39, 9], all while controlling for external factors and environmental variables. Few studies have explored the combination of visit duration and global pandemics but those that have are typically focused on the epidemiological facet. For instance, one study in Italy used data from Google Maps in an attempt to estimate the visit duration of individuals to certain places of interest in order to assess exposure risk to SARS-CoV-2. [44]. Other work [19] has demonstrated the value of mobile phone data for tracking where people tend to cluster though this has centered on geospatial coordinates rather than static place instances. The research presented in this work takes a next step, building on much of the research mentioned here to investigate the relationship between a change in visit duration due to the COVID-19 pandemic and the population characteristics of a city.

\section{Data}

Data collection for this work involved three steps. First, city boundaries were downloaded from the U.S. Census Bureau's TIGER Urban Areas dataset for the four most populated cities in the United States, namely New York City (NYC), Los Angeles (LA), Chicago (CHI), and Houston (HOU) [59]. Second, we used the Foursquare application programming interface [11] to access POI within each of the four cities throughout December 2019. This resulted in a set of POI for each city that included place names and addresses as attributes. This step was necessary as the Google Place Search API does not allow openended searches, but instead requires users to search for a specific place name or address. The Foursquare API allows place search given geographic coordinates and a radius. This was done multiple time at different locations in each city in order to collect as many POI as possible. Third, names and addresses from these four sets of POI were input into a thirdparty library [24] that accesses the Google Place Search API at two different times, namely January 2020 and January 2021. The results from these searches were two sets of Google POI for each of the four cities. The number of POI accessed for each city, at each query date, are shown in Table 1.

These POI include place name, latitude, longitude, place type, and visit duration as attributes. The place type attribute contained a comma delimited list of labels, e.g., Bar, Coffee Shop, Mexican Restaurant. The visit duration attribute consisted of two values, a lower 


\begin{tabular}{lrrr}
\hline City & January 2020 & January 2021 & Intersection \\
\hline New York City & 162,975 & 166,831 & 87,140 \\
Los Angeles & 191,010 & 193,490 & 100,855 \\
Chicago & 57,698 & 59,576 & 32,135 \\
Houston & 73,037 & 74,704 & 36,369 \\
\hline
\end{tabular}

Table 1: Number of places of interest for each city accessed in January 2020 and January 2021. The intersections of the two sets are also reported.

estimate and an upper estimate for visit duration in minutes. The precise methodology for how these bounds were determined is not made public, though Google Business Support states that "visit duration estimates are based on patterns of customer visits over the last several weeks" [18] and that "...we analyze aggregated and anonymized Location History data from people who have opted to turn this setting on from their Google Account" [34]. Notably, these data are contributed from anyone that has enabled Google's Location Services, regardless of the device's operating system. Of the POI accessed in January 2020, only $12.7 \%$ included values for the visit duration attribute. One year later, this percentage dropped to $8.9 \%$. Since visit duration requires a robust amount of historical data on which to report a time (while keeping individual visitors anonymous), this decrease in the visit duration attribute suggests a substantial drop in the actual place visits. Without further information pertaining to what the two values reported for visit duration represent, e.g., standard deviations from the mean, we re-assigned the visit duration attribute for each POI as a single value taken as the mean of the upper and lower bounds. We make the assumption that this value represents the average amount of time someone spends at a given POI.

Each POI is tagged with one or more place type labels (median of 2, mean of 3.5). The place type labels themselves range considerably given that they are contributed by establishment owners, managers, and visitors but supposedly curated by Google [16]. The average number of unique categories in each of the cities is 3,527. Restricting to only our intersection POI for each city, and only those with ten or more POI in each city, results in 888 unique place types. Analyzing this cleaned set of POI types, we discovered that the distribution of POI types is quite consistent across cities, despite the difference in overall POI counts. The Pearson's correlations between all pairs of city POI type distributions are all highly significant and above 0.92 in all cases, with NYC and HOU presenting the lowest correlation. Given average visit duration values for each POI in our datasets, we then grouped all POI by their place type label producing an array of visit durations for each place type in each city and for each of our two time periods. For clarity, Listing 1 provides an example of how these data are stored from a structural perspective.

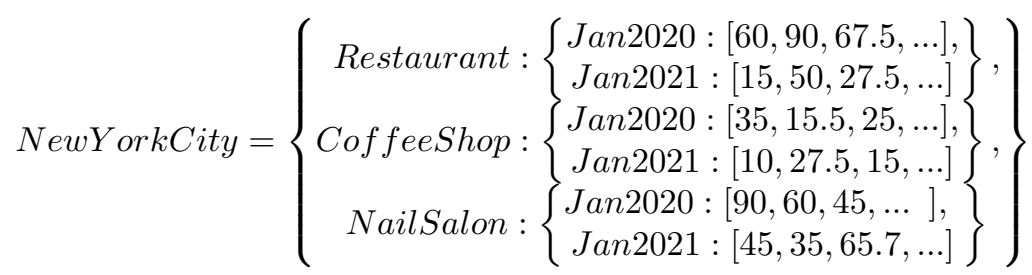


Demographic [62] and socio-economic [61] variables were obtained as 5-year estimates from the 2019 American Community Survey (ACS) for the census tracts in each city. Demographic variables included Percent Female, Percent White, Percent Black, Percent Asian and Percent Hispanic. Socio-economic variables included Percent Population 16 years and over in the labor force, Median Household Income (dollars), Median Family Income (dollars) and Percentage of families below the poverty line.

\section{Corroborating Google visit durations}

As mentioned previously, the visit duration data that we use in these analyses were accessed via Google Places, and very little information is available as to how the data were created. This leads to questions related to the validity of the data as well as the reproducibility of the research. To address these issues we compare the Google Places visit duration dataset with another visitation dataset, on which the process of collection and curation is public, namely SafeGraph's Patterns dataset [50]. SafeGraph collects visits to POI, including the average length of time someone spends at a POI through a large, anonymized panel of individuals that are tracked through various mobile applications [52]. While the underlying contributions and methods of data collection are different for SafeGraph and Google Places, both publish a version of POI visit duration through their platforms. Notably, the access conditions for these two platforms varies considerably. SafeGraph offers their data for research purposes allowing data storage for up to one year [51] whereas Google Places restricts downloading and throttles access to their content [15]. To identify the similarity between these two datasets, we first accessed SafeGraph's Core Places [49] dataset and downloaded all POIs in our four cities of interest. We then matched these POIs to those in the Google POI dataset. A match was identified if two POI had identical names and were within 100 meters of each other. This is an overly conservative matching technique but ensures a high degree of confidence that POI in two different datasets represent the same physical location. Table 2 shows the number of matches by city.

\begin{tabular}{lrr}
\hline City & POI Matches & Pearsons Correlation \\
\hline New York City & 2,031 & 0.345 \\
Los Angeles & 2,142 & 0.533 \\
Chicago & 1,926 & 0.352 \\
Houston & 1,422 & 0.215 \\
\hline \multicolumn{3}{c}{}
\end{tabular}

Table 2: Number of POI matches identified between SafeGraph Core Places and Google Places. Pearson's correlation is reported between a change in SafeGraph's Median Dwell Time and a change in Google Place's Visit Duration from January 2020 to January 2021.

For each POI in the SafeGraph dataset we accessed the median dwell time variable for each day in January 2020 and calculated the median, assigning a single value to each POI. We did the same for January 2021. We then took the difference between the two time periods as the change in dwell time. Finally, we calculated Pearson's correlation between the change in SafeGraph's dwell time and change in Google Place's visit duration, over the same time period. The results are shown in Table 2 and indicate that there are significant, positive correlations between the two datasets, in all four cities. Given the different methods of data collection, population sizes, and company-specific aggregation 
techniques, these results strongly indicate that there is validity to the Google Places visit duration data and further analysis using these data is warranted.

\section{Methodology}

The scripts and shareable data for all of the analysis presented in this paper are available in a public repository at https://github.com/ptal-io/VisitDuration. Figure 1 presents a graphical overview of the methodology used in this paper.
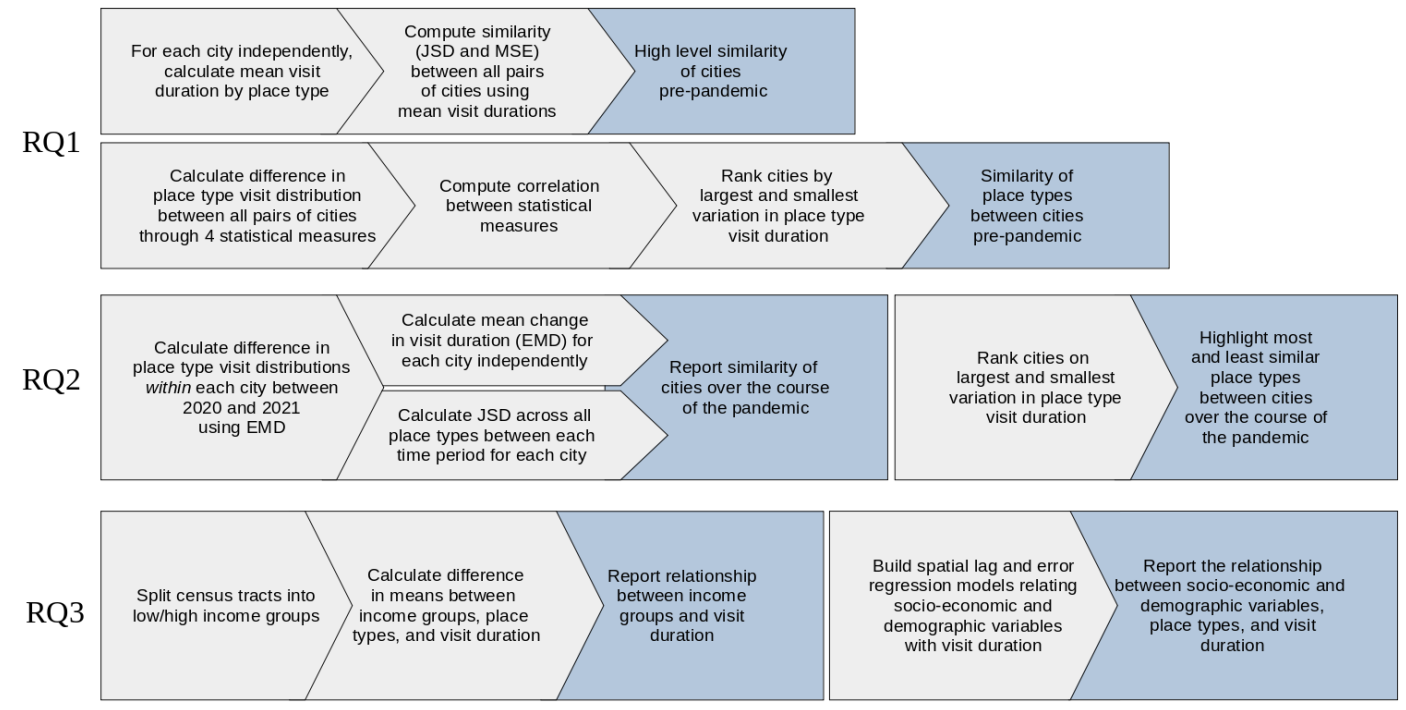

Figure 1: A graphical overview of the methodology workflow addressing each of the research questions (RQ).

\subsection{Between-city variation in visit duration}

The mean visit duration was calculated for each of the 888 place types in each of our four cities producing a discrete set of place type visit durations for each city. We then calculated the Jensen-Shannon Distance (JSD) between all pairs of cities. JSD is a method used to measure the dis-similarity between two discrete probability distributions, in this case our place type sets. The JSD calculation is shown in Equation 1 where $A$ and $B$ are two city place type visit duration distributions with $D$ being the relative entropy of $A$ to $B$ (Equation 2) where $M=\frac{1}{2}(A+B)$.

$$
\begin{aligned}
J S D(A \| B) & =\sqrt{\frac{D(A \| M)+D(B \| M)}{2}} \\
D(A \| M) & =\sum_{x \in \mathcal{X}} A(x) \log \left(\frac{A(x)}{M(x)}\right) .
\end{aligned}
$$


This method returns a single floating point value between 0 and 1 with 0 indicating identical sets and 1 complete dis-similarity. The results of this method indicate how similar two cities are with respect to their visit duration at the same place types. For comparison, we also calculated the mean square error between each pair of city visit duration sets.

\subsection{Between-place type variation in visit duration}

Next we explore individual place type visit durations by computing statistics that measure the difference between two distributions. Specifically, we calculated four different similarity measures.

As mentioned in Section 3, we aggregated all the visit durations from each POI instance and assign it as an array to it's place type, for each of our cities. We then calculated the mean visit duration for each place type in each city. Next, we subtracted the mean visit duration for a place type in one city, from the same place type in another city. In addition, we calculated the standard deviation to allow for comparison of variation in visit duration for place types between cities. A limitation of this difference-in-means approach is that it works best when the data are normally distributed, which is not always the case for our visit durations. To address this limitation, we performed a Kolmogorov-Sminorv (KS) test. A kernel density estimation was first applied to the visit duration arrays for each place type producing a set of place type distributions representing the visiting duration behavior. The K-S test was then used to calculate the maximum distance between two distributions represented as cumulative distribution functions. The test returns a significance value indicating the probability that the two sample distributions are drawn from the same population distribution. While not subject to the same normal distribution limitations, it does reduce assessment of similarity to a single (max) distance measure.

Next, we calculated the Earth Mover's Distance (EMD) between all cities. EMD measures the cost of changing one distribution into another. Cost is based on the amount of volume moved over a specific distance. This approach does not necessitate normal distributions nor does it report similarity based on a single maximum distance measure. The same place type distributions calculated for the K-S test were used as input to the EMD analysis. Finally, we calculated the difference between peak visit durations. The time where the frequency of visit durations reached a maximum was determined to be the peak. In the case where a maximum returned more than a single value, the time that returned the smallest difference between city place types was reported.

These four different approaches for calculating similarity were applied to all matching place types in all city pairs. We then calculated the correlation between the approaches across all cities. Finally, we ranked each city pair based on difference in average visit duration resulting in the most and least similar place type visiting behavior for each city pair in our dataset.

\subsection{Comparing pre-pandemic to during-pandemic}

To set a baseline, we first analyzed the pre-pandemic visit durations using the methods outlined above, with the goal of identifying differences between cities prior to the onset of pandemic mobility response and governmental policy action. We then re-ran the analysis on the data from January 2021, just under one year into the pandemic and observed the differences in results. 
Next, and to address $R Q 2$, we explicitly compared the change in visit duration before and during the pandemic, between cities. To accomplish this, we again turn to the Earth Mover's Distance method to provide a measure of similarity between two distributions of visit durations. First, we restricted our analysis to only those place types in the dataset that contained at least 20 places instances, during both time periods, and in all cities. This reduced our number of place types to 142 but ensured that there were enough data points on which to identify patterns and allow for robust comparison between time periods.

For each place type in a city, we calculated the EMD between the place type visit duration distribution in January 2020 and January 2021. We then took two approaches. First, we calculated the mean, median, and standard deviation of all the place type EMD values for a city and assigned it to that city. Second, we considered the range of place types instead of averaging across all of them. To accomplish this, we calculated the Jensen-Shannon Distance between the two time periods for each city, using the set of place type EMD values as input to our JSD. This allowed us to determine which cities are most and list similar based on the impact of the pandemic on place type visit behavior.

The visit duration attribute associated with each place instance is one piece of the puzzle however as it only reflects the POI that survived the first year of the pandemic. To provide context to the visit duration analysis we also investigated the change in number of POI associated with each place type in each of the cities to see which place types decreased or increased, and within which cities.

\subsection{Socio-economic and demographic factors}

In addressing $R Q 3$, we compare the change in visit duration over the one year time period with socio-economic and demographic characteristics of the communities in which the POI exist. Given the sparsity of some of the place types, for this analysis, we elected to focus on a subset of place types and selected those that we hypothesized might exhibit the largest and smallest changes in visit duration. Specifically, we chose to explore Restaurant, Coffee Shop, and Fuel station as well as all POI regardless of place type label.

As a first step, we investigated one specific socio-economic variable, namely income, and the difference between lower and higher income communities with respect to change in POI visit duration. We labeled each census tract in each of our cities as either lower income or higher income. Lower income communities are defined as those with a median household income below the United States median household income threshold of $\$ 63,179$ in 2018 [60]. Higher income communities are those above the median household income threshold. We calculated the mean change in visit duration for all POI in each census tract before the pandemic, and one year into the pandemic. Splitting census tracts into low income or high income groups, we then used Welch's two sample t-test to calculate the difference in means for each group, each place type, and each city.

Next, spatial error and spatial lag regression models that account for spatial dependence were adopted for all POI regardless of type as well as the three specific place types mentioned above, in each of the 4 cities. The dependent variable was the change in visit duration, coupled with our set of socio-economic and demographic independent variables. Spatial dependence was initially assessed and detected within a classical Ordinary Least Squares regression, using a range of diagnostics, such as Morans I, that indicated strong spatial autocorrelation of residuals, and Robust Lagrange Multiplier tests for error and

lag. For each city and POI type, we ran both spatial lag and error models designating

www.josis.org 
row-standardized spatial weights as minimum distances in which there would not be any neighborless observations.

\section{Results}

\subsection{Between-cities}

The results of our between-city variation in visit duration analysis based similarity analysis were a set of JSD dissimilarity values for each pair of cities. For the pre-pandemic dataset, the absolute JSD values were all low (0.0024 to 0.0058) indicating that overall, the cities are very similar. A JSD value of 0 indicates that two distributions are identical with a JSD of 1 indicating complete dissimilarity. To allow for better relative comparison, these values were normalized to between 0 and 1 (Table 3 ). The larger the number, the more dissimilar the cities are. The normalized mean square error of the difference in visit duration distributions is also reported.

\begin{tabular}{ll|rr|rrr}
\hline \multicolumn{2}{c}{ City Pairs } & \multicolumn{2}{c}{ January 2020 } & \multicolumn{2}{c}{ January 2021 } \\
\hline City A & City B & nJSD & nMSE & nJSD & nMSE \\
\hline New York City & Los Angeles & 0.41 & 0.64 & 0.47 & 0.57 \\
New York City & Chicago & 0.39 & 0.37 & 0.01 & 0.02 \\
New York City & Houston & 1.00 & 1.00 & 0.69 & 0.87 \\
Los Angeles & Chicago & 0.25 & 0.28 & 0.00 & 0.00 \\
Los Angeles & Houston & 0.00 & 0.00 & 1.00 & 1.00 \\
Chicago & Houston & 0.56 & 0.54 & 0.77 & 0.96 \\
\hline
\end{tabular}

Table 3: Normalized Jensen-Shannon Distance dissimilarity values and normalized mean square error for each pair of cities split by time period.

New York City is the most unique of the three cities, showing the highest degree of dissimilarity to the other three cities. In contrast, Los Angeles is most similar to the other three cities, on average. The most similar of our four focal cities are Houston and Los Angeles with Houston and New York City being the most dissimilar, based on our discrete set of place type visit duration values. These same patterns are also observed in the normalized mean square error analysis with only a slight increase in dissimilarity between New York City and Los Angeles, indicating this ranking of city similarity holds, irrespective of our chosen method of analysis.

The results of the same analysis on the January 2021 dataset suggests that the similarities between cities, as determined by place type visitation, changed substantially. While the absolute JSD values remained low (indicating high similarity), they almost doubled relative to the pre-pandemic JSD values with a range of $0.004-0.010$. Though Los Angeles and Houston were the most similar cities prior to the COVID-19 pandemic, they were determined to be the least similar cities 10 months into the pandemic. New York City became much more similar to Chicago and the similarity between New York City and Los Angeles remained consistent. Houston increased its dissimilarity to the other three cities, overall, becoming the most dissimilar city on average. As before, there is a strong correlation between the normalized JSD and the normalized means square error values. 


\subsection{Between-place types}

Next, we report on the similarities and differences between place types across cities based on the four different similarity approaches. Significant positive Pearson's correlation $(p<0.01)$ was observed between the results of the absolute difference in means, absolute difference in peak visitation, and EMD in both time periods. The pair of measures that produced the highest correlation were EMD and absolute difference in means (0.91) followed by EMD and absolute peak difference (0.49). There was no significant correlation found between the K-S statistic and any of the other three similarity measure. This is likely due to how different this approach is in identifying similarity, namely by measuring the largest difference between two cumulative distribution functions. In light of these results, future discussion will use EMD as the method through which we measure similarity between place type visit duration distributions.

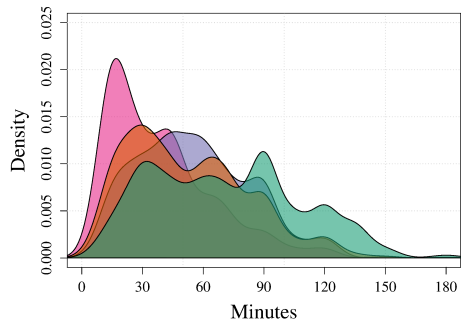

(a) Family Restaurant

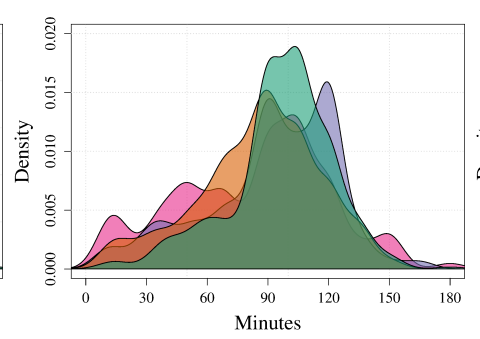

(b) Wine Bar

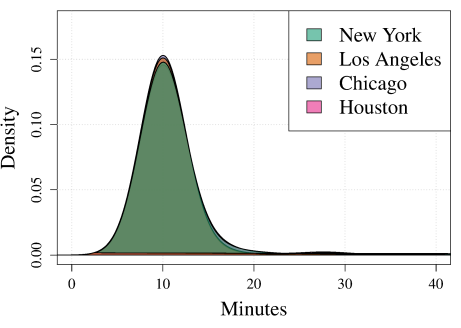

(c) Fuel Station

Figure 2: Density plots of visit duration for three place types in January 2020, prior to the COVID-19 pandemic.

Starting with the pre-pandemic dataset, the mean, median, and standard deviation of the pairwise city EMD were calculated for each place types. These descriptive statistics were then ranked, allowing us to determine which place types were most and least consistently different or similar, across all cities. Wine Bar, Family Restaurant, and Institute consistently appeared as the most regionally dissimilar place types prior to the pandemic. Two of the most dissimilar place types are shown in Figures $2 a$ and $2 b$. The most dissimilar distribution of visit durations were found for Family Restaurant, between New York City and Houston. A full list of place type and their corresponding EMD values are provided at https://github.com/ptal-io/VisitDuration.

Ranking each approach based on the average most similar place types across each city returned Fuel Station (Figure 2c) followed by Drug Store and Convenience Store. The single most similar distributions of visit duration were found in the place type Drug Stores between Chicago and Houston. From a broad category perspective, prior to the pandemic, Entertainment venues (e.g., Museums, Music Venues, Bars) tended to have the longest mean visit duration across all cities while everyday retail businesses (e.g., Fuel Stations, Convenience Stores, Liquor Stores) tended to have the shortest. The place type that most consistently reported the longest mean visit durations across all cities, prior to the pandemic, was Night Club, with Fuel Station again being the shortest.

We re-ran the analysis above for the POI dataset collected in January 2021, during the pandemic, and ranked place types based on averages across all cities. The place types that consistently reported the largest average EMD were Cocktail Bar (Figure 3b), Park, and a 


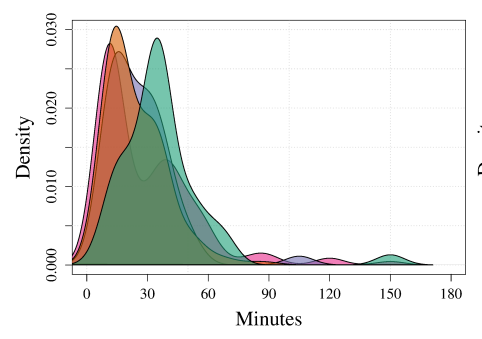

(a) Family Restaurant

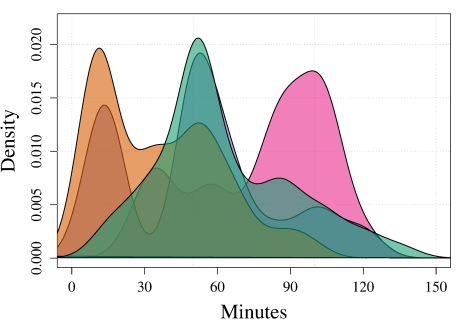

(b) Cocktail Bar

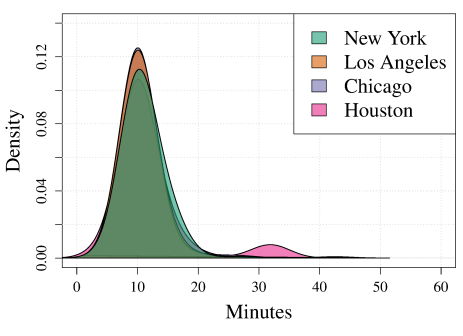

(c) Liquor Store

Figure 3: Density plots of visit duration for three place types in January 2021, during the third wave of the COVID-19 pandemic.

set of place types related to Salon (e.g., Nail, Hair, Beauty). Place types that were most varied prior to the pandemic, such as Family Restaurant, still appeared in the top dissimilar quintile but were not as dissimilar as others. In plotting the density estimations for Family Restaurant (Figure 3a), we observe a difference in the visit duration patterns between cities, but more interestingly, we see a substantial shift in the range of visit duration compared to the same place type pre-pandemic (Figure 2a). Notably, for this place type, the density plot for Houston has remained more or less consistent between the two time periods, whereas the other cities experienced more substantive changes. The place type with the largest regional difference in January 2021 was Cocktail Bar between Los Angeles and Houston. Sorting the set of place type EMD values from smallest to largest we discovered that the set of place types did not change much from pre-pandemic set. Everyday item business types such as Convenience Store, Liquor Store (Figure 3c), and Fuel Station were consistently similar between regions. The full set of January 2021 regional place type comparison values are provided at https://github.com/ptal-io/VisitDuration.

\subsection{Pre-pandemic to during-pandemic behavior}

Next, we calculated the EMD for each place type between the two time periods, for each city separately. Given an array of place type EMDs for each city, we first calculated the averages for each city, as mentioned in Section 4.3. The results indicate that the COVID-19 pandemic impacted cities in substantially different ways. As seen in Table 4, New York City reported the largest change with a mean EMD twice the value of Houston. Chicago and Los Angeles were closer to New York City with average EMD values of 11.26 and 10.97, respectively. The standard deviations for each city also highlight the amount of variability in change between place types. Again, the standard deviation of EMD values for Houston is half that of the other three cities. The results of the JSD analysis are similar with Houston being the city that is least similar to the other three. The most dissimilar pairs were Houston and New York City (JSD $=0.101$ ) and the most similar pair were Chicago and Los Angeles (JSD = 0.048). The city that was most similar on average to the other three cities was Chicago. These values speak to the overall similarities in how cities inhabitant's responded to the COVID-19 pandemic.

Next, we investigated a few select place types that we hypothesized were likely to show some of the most interesting changes over the one year time period, and examined the change in visit duration patterns between cities. Figure 4 presents the change in visit du- 


\begin{tabular}{lrrrr}
\hline & New York City & Los Angeles & Chicago & Houston \\
\hline Mean & 12.53 & 10.97 & 11.26 & 6.27 \\
Median & 7.11 & 6.02 & 6.70 & 4.36 \\
Standard Deviation & 12.59 & 12.44 & 12.46 & 5.96 \\
\hline
\end{tabular}

Table 4: Descriptive statistics for the place type Earth Mover's Distances between January 2020 and January 2021, split by city.

ration behavior for three place types, namely Bar, Coffee Shop, and Bank, in our four cities. In the Bar place type example we see substantial differences in the visit duration patterns of cites. In all cases, the bulk of visit duration decreased considerably but the amount of decrease is inconsistent across cities. The patterns for New York City, Los Angeles, and Chicago all demonstrate drastic changes whereas Houston split into two popular visit duration, one that peaks around 45 minutes and the other that stays consistent with the bulk of visit duration prior to the pandemic, peaking around 100 minutes. Purely looking at difference in mean visit duration for Bar, New York City, Los Angeles, and Chicago all reported decreases between 42.5 and 47.0 minutes, whereas Houston reported a mean change of 22.6 minutes. This trend continued for most of the place types with the largest decreases in visit duration at Restaurant, Tourist Attraction, and different Bar place types.

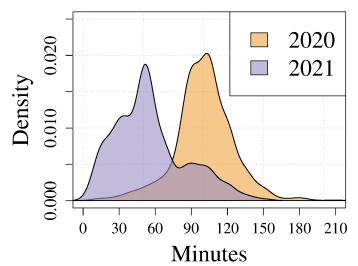

(a) Bar NYC

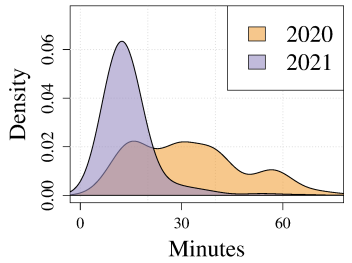

(e) Coffee Shop NYC

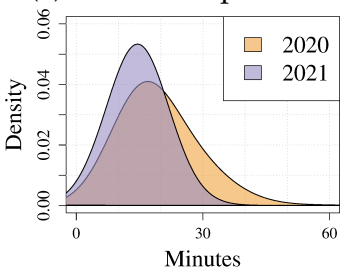

(i) Bank NYC

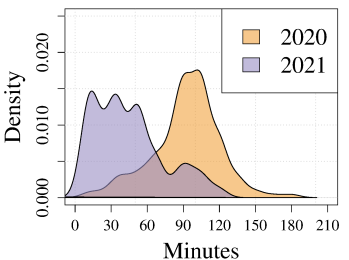

(b) Bar LA

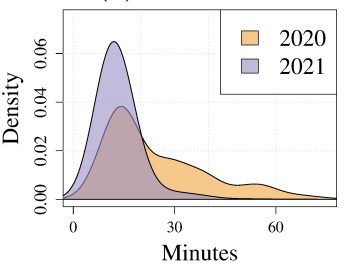

(f) Coffee Shop LA

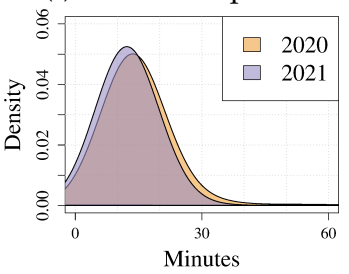

(j) Bank LA

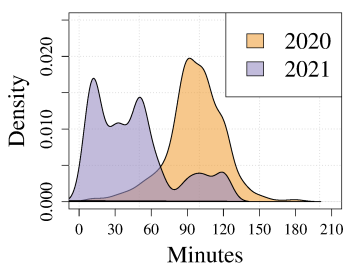

(c) Bar $\mathrm{CHI}$

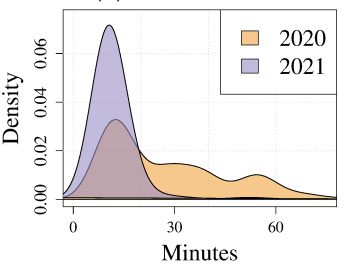

(g) Coffee Shop CHI

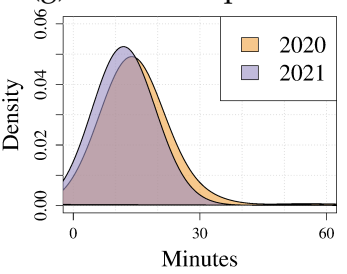

(k) Bank CHI

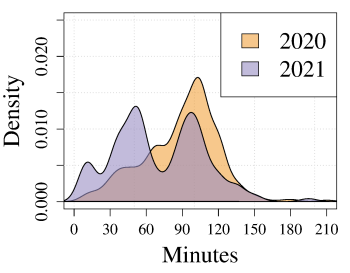

(d) Bar HOU

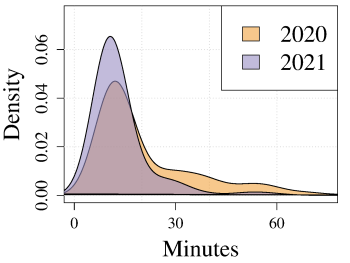

(h) Coffee Shop HOU

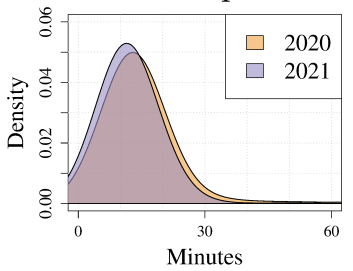

(l) Bank HOU

Figure 4: Density plots of visit duration for January 2020 and January 2021 split by city and place type. Bar (a-d), Coffee Shop (e-h), Bank (i-1). 
Coffee Shop (Figures 4e-h) reported a large number of visits that lasted between 15 and 60 minutes prior to the pandemic. During the pandemic, visit duration reduced considerably with all cities peaking around 15 minutes. Again, Houston showed the least amount of change but also showed the highest average peak at 15 minute duration prior to the pandemic. The Bank place type (Figures 4i-l) is shown here as an example of a place type that changed very little between time periods, and across cities. New York City showed the largest change due to slightly longer visit durations prior to the pandemic. In all other cities, the average density of visit duration remained almost exactly the same between the two time periods.

Stepping back from the visit duration values themselves, we examine the change in place type tags associated with cities over our one year time period. In this analysis, we look at all POI in each year, regardless of whether or not the place instances intersected. The place types that experienced the largest change (increase and decrease) are shown in Table 5. For instance, the number of places tagged as Delivery Restaurant increased by an average of $202 \%$ across all cities of interest. This ranged from $521 \%$ in New York City to just under 17\% in Houston. Other top place types included Mailing and Shipping Services as well as Liquor Store, and Supermarket. A number of place type tags also decreased over the course of the year. The number of gyms decreased by $88 \%$ on average across all cities followed by a number of place types related to beauty services as well as entertainment establishments that serve liquor.

\begin{tabular}{lrrrr|lrrrr}
\hline \multicolumn{3}{c}{ Largest Increase (\%) } \\
\hline Category & NYC & LA & CHI & HOU & Category & NYC & LA & CHI & HOU \\
\hline Delivery Restaurant & 521.2 & 91.5 & 179.7 & 16.9 & Gym & -89.6 & -94.0 & -85.8 & -82.9 \\
Mailing Service & 317.5 & 94.1 & 125.0 & 46.2 & Nail Salon & -81.8 & -91.2 & -81.3 & -74.6 \\
Liquor Store & 183.7 & 32.7 & 8.6 & 5.8 & Salad Shop & -68.7 & -74.4 & -84.7 & -82.9 \\
Electronics Parts Store & 93.5 & 29.8 & 17.3 & 22.5 & Beauty Salon & -75.6 & -85.3 & -76.9 & -67.6 \\
Wine Store & 131.0 & 3.7 & 3.2 & 20.8 & Hair Salon & -77.7 & -87.4 & -75.0 & -61.0 \\
Supermarket & 59.8 & 16.6 & 30.3 & 42.6 & Dentist & -66.4 & -77.6 & -64.4 & -72.4 \\
Mailbox Rental & 87.1 & 14.4 & 45.8 & 2.0 & Ice Cream Shop & -66.8 & -70.7 & -69.7 & -65.6 \\
Freight Forwarding & 79.3 & 16.8 & 47.8 & 2.0 & Cocktail Bar & -76.9 & -69.9 & -76.2 & -48.8 \\
Business Center & 69.6 & 11.2 & 31.0 & 10.0 & Barber Shop & -59.6 & -75.9 & -75.6 & -60.4 \\
Takeout Restaurant & 93.2 & -2.8 & 54.3 & -12.4 & Bar & -73.5 & -57.0 & -70.0 & -38.5 \\
\hline
\end{tabular}

Table 5: Place types that saw the largest percentage increase and decrease over the one year time period.

Restricting our dataset to only those place instances that existed in both time periods, we explored the change in place type labels. Whereas some places added place type tags, others removed them. On average there was a $155.0 \%$ increase in the number of existing places of interest that added the place type Delivery Restaurant across all cities ranging from $360.2 \%$ in New York City to 33.3\% in Houston. Other leading tags included Mailing Services, Supermarket, and Fast Food Restaurant. Place type tags that were most often removed from place instances were Corporate Office (-68.1\%), Salad Shop, and Tea House. While it is to be expected that places of interest might change their tags over the course of a typical year, the dramatic decrease or increase in these types of place labels can, at least partially, be attributed to the pandemic. 


\subsection{Community socio-economic and demographic characteristics}

The results of the low and high income community analysis are shown in Table 6. Each value is the average minute decrease in visit duration from the start of the pandemic to one year later. Each cell in the table contains the minutes averaged across all low income communities first followed by minutes average across all high income communities.

\begin{tabular}{lllll}
\hline & All POI & Restaurant & Coffee Shop & Fuel Station \\
\hline New York City & $12.01 / 12.65$ & $16.43 / 18.45^{* * *}$ & $12.99 / 15.94^{* * *}$ & $0 / 0.54^{* *}$ \\
Los Angeles & $9.69 / 10.86^{* *}$ & $16.31 / 19.39^{* * *}$ & $9.94 / 11.65^{* *}$ & $0.24 /-0.07$ \\
Chicago & $9.61 / 13.72^{* * *}$ & $12.85 / 20.36^{* * *}$ & $7.75 / 14.76^{* * *}$ & $0.53 / 0.12$ \\
Houston & $5.51 / 6.05^{*}$ & $6.85 / 8.34^{* * *}$ & $4.34 / 5.97^{* * *}$ & $0.73 / 0.09^{* *}$ \\
\hline${ }^{*} \mathrm{p}<0.1{ }^{* *} \mathrm{p}<0.05,{ }^{* * *} \mathrm{p}<0.01$. & & &
\end{tabular}

Table 6: Average minute decrease in visit duration between January 2020 and January 2021 split by low income and high income communities (low/high).

In all cases but Fuel Station, the change in visit duration was greater in higher income communities than in lower income communities between the start of the pandemic and during the pandemic. The differences in Restaurant visit duration were significant in all cities with Chicago showing the largest difference in visit duration. All POI including three place types (other than Fuel Station) were significant for Los Angeles and Chicago. New York City and Houston demonstrated significance in some categories but not others. As identified in the previous analysis, the change in visit duration for Houston was substantially lower for both low and high income communities than the other three cities. The difference in visit duration for Fuel Station are all quite low, both for high income and lower income communities, across all cities. While there is a significant difference in some cases, the generally low values suggest that there really is very little difference in Fuel Station visiting behavior before and during the pandemic, and between low and high income communities.

The results of the spatial error regression models were strikingly similar to those of the spatial lag models. Over all subsets of the data, the spatial error model demonstrated the best performance (Akaike info criterion, Log likelihood, pseudo- $R^{2}$ ) in most, but not all, cases. In order to compare results across cities and place types, we elected to report the results of the spatial error regression models for the remainder of this work. The pseudo- $R^{2}$ values for each of these regression models were quite low (0.007 - 0.176) indicating that the socio-economic and demographic variables selected in our model did a poor job, overall, in explaining the difference in visit duration before and during the pandemic. The purpose of this work, however, was not to fully explain the change in visit duration between census tracts, but rather determine which of our chosen socio-economic and demographic variables most contributed to a change in visit duration. More specifically, we identified which combination of independent variables significantly contributed to a change in the dependent variable and whether this change was positive or negative.

The full results of the spatial error regression models are shown in Tables 7-10 in the Appendix. While there is no consistent agreement of significance across all cities and all place types, some commonalities were identified. Percentage of Black and Hispanic population as well as Percentage of the population 16 years of age or higher in the labor force tended

www.josis.org 
to contribute the most to the dependent variable across all cities, whereas percentage White and Asian were the least impactful of the variables. In all cases where the contribution of the variables representing percentage Black or Hispanic are significant, the value is always negative. This means that for each increase in percentage of that race in a census tract, the difference in visit duration between the start of the pandemic and one year later, decreases. Female gender was significant for all POI in New York City, Los Angeles, and Chicago but was negative in Los Angeles and Chicago, and positive in New York City. The percentage of the population 16 years of age or older in the labor force was significant and positive for Restaurant and Coffee Shop (New York City and Los Angeles) suggesting that census tracts with older populations also had a larger decrease in visit duration over the course of the pandemic, than those with young populations.

On average, the regression models built on the Restaurant place types reported the most number of significant coefficients while change in visit duration to Fuel Station were least explained by the chosen variables. In fact, only the variables in the Chicago dataset displayed significance with Percentage White, Black, and Hispanic all showing a slightly significant positive relationship with increase change in visit duration. Of the four cities in our analysis, the regression models built on data from Houston explained the change in visit duration the least, reporting no significant coefficients for All POI, or the Fuel Station place type.

\section{Discussion}

In this section, we revisit the original research questions presented in the introduction and provide responses based on the interpreted results of our analysis. In responding to $R Q 1$, the results indicate that there were significant differences in place type visit duration both in January 2020, prior to the pandemic, as well as January 2021, during the third wave of the pandemic in the United States. Our results indicate that while there was some general agreement between cities on which place types had longer visit durations than others, e.g., Restaurant vs. Fuel Station, there are important differences between cities. For instance, on average, prior to the pandemic, there was almost a 30 minute difference in visit duration to Wine Bars between New York City and Los Angeles. During the third wave of the pandemic, this difference decreased to 18 minutes and the visit durations within all of the cities changed. Using a variety of statistical techniques, we demonstrated numerous ways in which the visiting behavior of individuals to place types varies across regions in the United States. This aligns with existing research concerning variation in when people choose to visit places [37] and indicates that, on average, inhabitants in difference cities interact with places within their cities in a range of unique ways.

These findings will be of interest to urban planners and policy makers. The results provide a better understanding of the interaction between place types and, in combination with time of day and day of the week behavior, these visit duration values can be used to determine where resources should be allocated within a city. Planners developing a new city or performing a revitalization project may look at another city they know to be similar to their own in order to predict what the addition of a new park, or supermarket might do to the human activity patterns within their city. From a computational perspective, this is useful information as well. Companies operating geospatial search and information applications such as Google Maps need to index attribute information such as visit dura- 
tion. Doing so for each region and subregion throughout the world requires a substantial amount of space and processing power. The results of this work indicate that while the visit duration of some place types vary considerably between regions, others, e.g., Fuel Stations, do not. The place types that vary the least could be stored and indexed once and applied to a larger administrative region whereas highly variable places types, e.g., Family Restaurants, would need to be indexed at a local, higher resolution.

In response to $R Q 2$, our results indicate that there is a quantifiable difference in place type visit duration before the pandemic and one year later. Our analysis presented in Section 4.3 uses a robust approach to quantify the difference between cities as a whole over the one year time span, as well as individual place type visit durations within each city. New York City reported the highest degree of variability in place type visit durations between the two time periods, followed by Chicago and Los Angeles. Houston was a distant fourth, reporting the least amount of change over the initial months of the pandemic. The reasons for this are complex, likely involving both social and political factors. Though a full examination of this topic is outside of the scope of this paper, it has been widely reported that officials from the State of Texas, of which Houston is the largest city, were more hesitant to adopt social distancing measures and face coverings than other states [40,53]. Texas' policies related to COVID-19 response were some of the least restrictive in the country [42], much less than the other three cities in our analysis. This at least partially explains the lack of change in aggregated place type visit durations in Houston relative to the other cities in our analysis.

At the individual place type resolution, we again see that there is regional variation in visit duration before and during the pandemic. Some place types such as Bar experienced a significant reduction in visit duration in cities like New York City and Chicago, with less of a change in Houston. For this specific place type we saw two peaks emerge in 2021, one with the same visit duration as pre-pandemic times, and one with a much shorter visit duration. In all cases except for Houston, the shorter visit duration peak was substantially larger. This again exemplifies the difference in how inhabitants of each city responded to the pandemic. Other place types such as Bank saw little to no change over the course of a year, regardless of the city. These results are a first step towards a pandemicrelated resilience measure [48] demonstrating that some place types are more resilient than others, and that resilience is often a regionally variant concept. Overall, the place types that exhibited the least amount of variance over the course of the pandemic tended to be related to essential services such as Fuel Station, Bank, Supermarket. Non-essential services or leisure types tended to be both the most temporally variant (over the course of one year) and spatially variant with place types such as Bar, Beauty Salon, Restaurant showing the largest variation. This again is useful information to urban planners in determining which place types support consistent human activity behavior regardless of a global pandemic and which are most susceptible. This is also useful to public health officials and epidemiologists attempting to identify place types with consistently long visit durations that may facilitate the spread of a virus.

The results of this analysis also highlight some of the ways in which business owners responded to the COVID-19 pandemic. There was a notable increase in certain place types such as Delivery Restaurant and Mailing Service. In some cases, places of interest that existed prior to the pandemic updated their Google Place profile to include Delivery Restaurant explicitly in response to dine-in restrictions. Many other businesses were shuttered either due to local government enacted policies, or a fear-induced decline in business. Our find- 
ings reflect the antidotal information reported by news agencies [5] with place types such as Gym, Nail Salon, and Bar experiencing significant decreases over the course of the early pandemic.

Lastly, we addressed $R Q 3$ by investigating the relationship between place type visit duration and the socio-economic and demographic characteristics of the communities in which places are situated. Through our analysis we discovered that, again, there was regional variation in the relationship between the characteristics of a population and the visit duration to POI, and that this variation is present across place types as well. We found that places of interest in lower income communities were statistically more likely to see a smaller decrease in visit duration than those in higher income communities. What this means is that those in more affluent communities were more likely to react to the pandemic by decreasing the time that they spent at a place. While there are multiple factors driving this reaction, one could argue that populations in lower income communities either did not have the option to change their behavior (e.g., they could not afford to not work or buy food), were not as informed as more affluent communities, or they simply elected not to react in the same way. Our analysis also found that communities with high percentages of Black or Hispanic populations were more likely, on average, to experience less of a decrease in visit durations. Communities with a higher proportion of population 16 and over in the labor force also also tended to show a larger decrease in visit duration. All of these findings indicate that the pandemic did not affect populations equally and correspond with existing research in this domain. Public health agencies, government assistance programs, and aid organizations should be made aware of these findings as this provides evidence on which to determine where additional funding and education efforts should be made during a pandemic.

As with the previous analysis, we observed regional variation with respect to the relationship between population characteristics and place type visit duration. While a number of the community socio-economic and demographic characteristics were significantly linked to a change in visit duration in a city like New York City, Houston reported far few significant variables. This can partially be explained by the smaller change in visit duration over the one year period, as well as the State's limited pandemic response. Based on the results, one could also argue that a city such as Houston just has less variation in how different populations responded to the pandemic. Again, much of this may be explained through the social, climatic, and political differences between regions within the U.S., but we must acknowledge that a change in place type visit duration was not consistently linked with certain socio-economic and demographic variables in all cities.

\subsection{Limitations}

The method through which Google collects, curates, and publishes the visit duration values are abstracted from anyone outside of the company. Because of this, the process cannot be scrutinized and we must assume that the published visit duration values are appropriate for the type of analysis performed here. Efforts have been made in this work to determine the validity of this data by comparing it to an additional source, namely SafeGraph. The biases associated with Google Places dataset as a whole must also be acknowledge as a limitation. While Google Maps, and by extension Google Places, is the largest source of POI data available today [17] the visitation data that they collect is not exhaustive and 
represents a sample of the population that use their services. The findings presented in this paper should be interpreted within this context.

During our data collection, we observed from the intersection of POI accessed between the two dates, that an average of $50 \%$ of POI are accessible between the two time periods. A portion of this may be explained by our data collection methods, but the underlying Foursquare POI, on which the Google Places were queries, remained the same. Two possible reasons for the limited amount of overlap are that (a) Places permanently or temporarily closed between our two data access times, or (b) The Google Places platform removed these places for a reason unbeknownst to us. Given the significant reduction in POI, we assume that many places closed between the two time periods, likely as a result of the pandemic. With respect to the POI attributes, the fact that many POI are assigned multiple place types means that the visit duration of a single POI will likely contribute to two or more place type visit duration values. We do not believe this is a significant issue with our analysis but rather speaks to the reality of place type taxonomies and that most places do not cleanly fit into a single type.

In exploring the relationship between the socio-economic and demographic variables to our aggregate place type visit duration values, census tracts were chosen as the geography for analysis. While this is a common dataset used in such analysis it does have limitations. Census tracts were designed with the goal of reporting population characteristics within regions with similar population counts. This, however, means they vary substantially in geography area. Census tracts may also span multiple neighborhoods, which are harder to define with boundaries, but tend to have more cohesive demographic traits. Our analysis assumes that visitors to places are only from the census tract in which the place exists. We know this is not always the case, especially with certain types of places that are destinations for people from all over a city, e.g., upscale restaurant. This issue could be approached in future work by incorporating a distance decay weighting or addition knowledge from surveying city inhabitants.

\subsection{Future work}

Future work on this topic will take a number of different forms. The current analysis explores four U.S cities over a one year time period. Future analysis will incorporate additional cities of different sizes and demographics in multiple countries. The time period will be extended to observe any changes in visit duration over the course of the pandemic and into pandemic recovery. Though hours of operation and popular visitation times have been analyzed in previous research, further analysis will incorporate visit duration with these patterns to gain a more holistic understanding of the differences in visit behavior.

From a population characteristics perspective, future work will incorporate additional socio-economic, demographic, and environmental variables and study the effects of changing the geographic resolution on which these variables are aggregated. This would allow us to determine the robustness of our findings across different spatial scales and provide evidence on which to inform various levels of government, e.g., local vs. national. Surveying visitors to establishments would also allow us to better understand what factors lead to any change in visit duration as the data we present here does not present motivation for a change in behavior.

The semantics involved in assigning place type labels is particularly interesting as well. What is a wine bar?, for instance. While in this case, owners and operators of establishments

www.josis.org 
have added their own place type labels to their Google profiles, it does not mean that everyone agrees with the chosen place types. There are likely regional differences in how people choose to label places. This is in and of itself an interesting topic and supports the need for further place-based regional activity analysis. In addition, further work may explore the susceptibility of place types to label changes. For instance, which places could more easily change their type in response to a global pandemic.

\section{Conclusions}

Knowing the length of time that someone spends at a place is useful in designing cities, determining zoning regulations, and funding infrastructure projects. The fact that visit duration varies between place types is common knowledge but the degree to which this differs between cities is less known and can be useful for understanding a city's population and informing public policy. Identifying the ways in which visitation patterns change due to a global pandemic tells us a lot about a region and its inhabitants. It forms the basis on which to better understand a population's resilience and susceptibility of certain activities to regional variation. In this work, we identified a number of ways that place type visit durations differ (and do not differ) between U.S. cities. We show how these patterns were changed by the onset of the COVID-19 pandemic and measure city response through the observed changes in these place type visit durations. An analysis of each city's population characteristics demonstrated that not all Google Places visitors responded to the pandemic in the same way and that, in general, younger, lower income, Black and Hispanic visitors were less likely to respond to the COVID-19 pandemic through a reduction in place visit duration. Though little existing work has explored the nuanced changes and regional differences in visit duration due to the COVID-19 pandemic, this work complements existing research related to the impact of COVID-19 on certain subsets of the U.S. populations. The findings of this research provide actionable insight related to the inequities of the pandemic with the goal of informing policy makers and the public.

\section{Acknowledgments}

This research was supported in part by the Natural Sciences and Engineering Research Council of Canada (NSERC) and the Population Studies and Training Center at Brown University (through the generosity of the Eunice Kennedy Shriver National Institute of Child Health and Human Development [P2C HD041020]).

\section{References}

[1] Benitez, J., Courtemanche, C., And Yelowitz, A. Racial and ethnic disparities in covid-19: evidence from six large cities. Journal of Economics, Race, and Policy 3, 4 (2020), 243-261. doi:10.1007/s41996-020-00068-9.

[2] Chande, A., Lee, S., Harris, M., Nguyen, Q., Beckett, S. J., Hilley, T., ANDRIS, C., AND WEITZ, J. S. Real-time, interactive website for US-county-level COVID-19 event risk assessment. Nature Human Behaviour 4, 12 (2020), 1313-1319. doi:10.1038/s41562-020-01000-9. 
[3] Chang, S., Pierson, E., KoH, P. W., Gerardin, J., Redbird, B., Grusky, D., AND LESKOVEC, J. Mobility network models of covid-19 explain inequities and inform reopening. Nature 589, 7840 (2021), 82-87. doi:10.1038/s41586-020-2923-3.

[4] Chen, L. M., Farwell, W. R., And Jha, A. K. Primary care visit duration and quality: does good care take longer? Archives of internal medicine 169, 20 (2009), 18661872. doi:10.1001/archinternmed.2009.341.

[5] CHIU, A. The fitness industry is trying to lure gym members back - but experts say it's using flawed data. Washington Post (Sep 2020).

[6] Chon, Y., Lane, N. D., Li, F., Cha, H., And ZHAO, F. Automatically characterizing places with opportunistic crowdsensing using smartphones. In Proceedings of the 2012 ACM conference on ubiquitous computing (2012), pp. 481-490. doi:10.1145/2370216.2370288.

[7] Dall'Alba, R., AND RochA, D. G. Brazil's response to COVID-19: commercial determinants of health and regional inequities matter. The Lancet Global Health (2021). doi:10.1016/S2214-109X(21)00146-7.

[8] De FreitAs, C. Weather and place-based human behavior: recreational preferences and sensitivity. International journal of biometeorology 59, 1 (2015), 55-63. doi:10.1007/s00484-014-0824-6.

[9] De Negri, F., Galiezz, R., Miranda, P., Koeller, P., Zucoloto, G., Costa, J., FARIAS, C., Travassos, G., AND MEDRONHO, R. Socioeconomic factors and the probability of death by Covid-19 in Brazil. Journal of Public Health (Oxford, England) (2021). doi:10.1093/pubmed/fdaa279.

[10] De VRIES, J., RoY, D., AND DE KOSTER, R. Worth the wait? how restaurant waiting time influences customer behavior and revenue. Journal of operations Management 63 (2018), 59-78. doi:10.1016/j.jom.2018.05.001.

[11] FOURSQUARE. Foursquare developer documentation, Aug 2020. [Online; accessed 6. Aug. 2021], https:/ / developer.foursquare.com.

[12] Franch-Pardo, I., Desjardins, M. R., Barea-Navarro, I., ANd Cerdè, A. A review of GIS methodologies to analyze the dynamics of COVID-19 in the second half of 2020. Transactions in GIS (2021). doi:10.1111/tgis.12792.

[13] GAO, S., JANOWICZ, K., AND COUCLELIS, H. Extracting urban functional regions from points of interest and human activities on location-based social networks. Transactions in GIS 21, 3 (2017), 446-467. doi:10.1111/tgis.12289.

[14] GAO, S., RAO, J., KANG, Y., LIANG, Y., AND KRUSE, J. Mapping county-level mobility pattern changes in the United States in response to COVID-19. SIGSpatial Special 12, 1 (2020), 16-26. doi:10.1145/3404820.3404824.

[15] Google, Inc. Google Maps Platform Terms Of Service | Google Cloud, May 2020. [Online; accessed 11. Nov. 2021], https://cloud.google.com/maps-platform/terms. 
[16] Google, InC. Guidelines for representing your business on google. Tech. rep., Google, 2021. https://support.google.com/business/answer/3038177/.

[17] Google, INC. Places | Google Maps Platform | Google Cloud. Tech. rep., Aug 2021. [Online; accessed 3. Aug. 2021], https://cloud.google.com/maps-platform/places.

[18] Google, INC. Popular times, wait times, and visit duration. Tech. rep., Google, 2021. https://support.google.com/business/answer/6263531?hl=en.

[19] Grantz, K. H., Meredith, H. R., Cummings, D. A., Metcalf, C. J. E., Grenfell, B. T., Giles, J. R., Mehta, S., Solomon, S., Labrique, A., Kishore, N., ET AL. The use of mobile phone data to inform analysis of covid-19 pandemic epidemiology. Nature communications 11, 1 (2020), 1-8. doi:10.1038/s41467-020-18190-5.

[20] Hamstead, Z. A., Fisher, D., Ilieva, R. T., Wood, S. A., McPhearson, T., AND KREMER, P. Geolocated social media as a rapid indicator of park visitation and equitable park access. Computers, Environment and Urban Systems 72 (2018), 38-50. doi:10.1016/j.compenvurbsys.2018.01.007.

[21] HaRdY, B. L., AND LOGAN, T. D. Racial economic inequality amid the COVID-19 crisis. The Hamilton Project (August 2020).

[22] Honey-Rosés, J., Anguelovski, I., Chireh, V. K., Daher, C., Konijnendijk VAN Den Bosch, C., LitT, J. S., MaWani, V., McCall, M. K., Orellana, A., OSCILOWICZ, E., ET AL. The impact of covid-19 on public space: an early review of the emerging questions-design, perceptions and inequities. Cities $\mathcal{E}$ Health (2020), 1-17. doi:10.1080/23748834.2020.1780074.

[23] Huifeng, P., AND HA, H.-Y. Temporal effects of online customer reviews on restaurant visit intention: the role of perceived risk. Journal of Hospitality Marketing $\mathcal{E}$ Management (2021), 1-20. doi:10.1080/19368623.2021.1897053.

[24] JAin, A. Popular Times GitHub repository, 2018. [Online; accessed 6. Aug. 2021], https://github.com/apollojain/popular_times.

[25] KANG, C., MA, X., TONG, D., AND LiU, Y. Intra-urban human mobility patterns: An urban morphology perspective. Physica A: Statistical Mechanics and its Applications 391, 4 (2012), 1702-1717. doi:10.1016/j.physa.2011.11.005.

[26] KIM, H.-M., AND KWAN, M.-P. Space-time accessibility measures: A geocomputational algorithm with a focus on the feasible opportunity set and possible activity duration. Journal of geographical Systems 5, 1 (2003), 71-91. doi:10.1007/s101090300104.

[27] Kimes, S. E., AND RobSON, S. K. The impact of restaurant table characteristics on meal duration and spending. Cornell Hotel and Restaurant Administration Quarterly 45, 4 (2004), 333-346. doi:10.1177/0010880404270063.

[28] Kimes, S. E., WirTZ, J., AND NoOne, B. M. How long should dinner take? measuring expected meal duration for restaurant revenue management. Journal of Revenue and Pricing Management 1, 3 (2002), 220-233. doi:10.1057/palgrave.rpm.5170026. 
[29] Krumm, J., Rouhana, D., And Chang, M.-W. Placer++: Semantic place labels beyond the visit. In 2015 IEEE International Conference on Pervasive Computing and Communications (PerCom) (2015), IEEE, pp. 11-19. doi:10.1109/PERCOM.2015.7146504.

[30] Lim, K. H., Chan, J., Leckie, C., And Karunasekera, S. Personalized tour recommendation based on user interests and points of interest visit durations. In Twenty-Fourth International Joint Conference on Artificial Intelligence (2015), AAAI Press, pp. 1778-1784. doi:10.5555/2832415.2832496.

[31] LiU, X., ANDRIS, C., AND RAHIMI, S. Place niche and its regional variability: Measuring spatial context patterns for points of interest with representation learning. Computers, Environment and Urban Systems 75 (2019), 146-160. doi:10.1016/j.compenvurbsys.2019.01.011.

[32] LiU, Y., WEI, W., SUN, A., AND MiAO, C. Exploiting geographical neighborhood characteristics for location recommendation. In Proceedings of the 23rd ACM international conference on conference on information and knowledge management (2014), pp. 739748. doi:10.1145/2661829.2662002.

[33] Massimo, D., AND RICCI, F. Next-POI recommendations matching users visit behaviour. In Information and Communication Technologies in Tourism 2021. Springer, 2021, pp. $45-57$.

[34] Matt D'Zmura. Behind the scenes: popular times and live busyness information. Tech. rep., Google, 2020. https://blog.google/products/maps/maps101-populartimes-and-live-busyness-information/.

[35] McKenZIE, G., AND ADAMS, B. A country comparison of place-based activity response to COVID-19 policies. Applied Geography 125 (2020), 102363. doi:10.1016/j.apgeog.2020.102363.

[36] McKenZie, G., AND JAnOwicz, K. Where is also about time: A locationdistortion model to improve reverse geocoding using behavior-driven temporal semantic signatures. Computers, Environment and Urban Systems 54 (2015), 1-13. doi:10.1016/j.compenvurbsys.2015.05.003.

[37] McKenzie, G., Janowicz, K., GaO, S., And Gong, L. How where is when? On the regional variability and resolution of geosocial temporal signatures for points of interest. Computers, Environment and Urban Systems 54 (2015), 336-346. doi:10.1016/j.compenvurbsys.2015.10.002.

[38] MCKenzie, G., AND ROMM, D. Measuring urban regional similarity through mobility signatures. Computers, Environment and Urban Systems 89 (2021), 101684. doi:10.1016/j.compenvurbsys.2021.101684.

[39] MCLaren, J. Racial disparity in COVID-19 deaths: Seeking economic roots with census data. The BE Journal of Economic Analysis \& Policy (2021). doi:10.1515/bejeap2020-0371.

[40] Mervosh, S., BAKer, M., Mazzei, P., AND Walker, M. One Year, 400,000 Coronavirus Deaths: How the U.S. Guaranteed Its Own Failure. The New York Times (Mar 2021).

www.josis.org 
[41] Miller, H. J. Modelling accessibility using space-time prism concepts within geographical information systems. International Journal of Geographical Information System 5, 3 (1991), 287-301. doi:10.1080/02693799108927856.

[42] New YORK Times. See Reopening Plans and Mask Mandates for All 50 States, Jul 2021. https:/ / www.nytimes.com/interactive/2020/us/states-reopen-mapcoronavirus.html.

[43] Noulas, A., Scellato, S., Lathia, N., AND Mascolo, C. A random walk around the city: New venue recommendation in location-based social networks. In 2012 International Conference on Privacy, Security, Risk and Trust and 2012 International Confernece on Social Computing (2012), Ieee, pp. 144-153. doi:10.1109/SocialCom-PASSAT.2012.70.

[44] Oliva, C., and Favato, G. Risk of exposure to covid-19: Visit duration data can inform our daily activities choices: An epidemiological investigation using community mobility data from the metropolitan area of genoa, italy. International Journal of Environmental Research and Public Health 18, 9 (2021), 4632. doi:10.3390/ijerph18094632.

[45] PAN, Y., Zeng, W., GuAN, Q., YAO, Y., LiAng, X., Yue, H., Zhai, Y., AND WANG, J. Spatiotemporal dynamics and the contributing factors of residential vacancy at a fine scale: A perspective from municipal water consumption. Cities 103 (2020), 102745. doi:10.1016/j.cities.2020.102745.

[46] Parrish II, R. C., Menendez, M. E., Mudgal, C. S., Jupiter, J. B., Chen, N. C., AND RING, D. Patient satisfaction and its relation to perceived visit duration with a hand surgeon. The Journal of hand surgery 41, 2 (2016), 257-262. doi:10.1016/j.jhsa.2015.11.015.

[47] Ristea, A., Al Boni, M., Resch, B., Gerber, M. S., And Leitner, M. Spatial crime distribution and prediction for sporting events using social media. International Journal of Geographical Information Science 34, 9 (2020), 1708-1739. doi:10.1080/13658816.2020.1719495.

[48] Rockefeller Foundation. City resilience framework. Tech. rep., December 2015. https://www.rockefellerfoundation.org/report/city-resilience-framework/.

[49] SafeGraph. Core Places, Oct 2021. [Online; accessed 25. Oct. 2021], https://docs.safegraph.com/docs/core-places.

[50] SafeGraph. Patterns | SafeGraph Docs, Oct 2021. [Online; accessed 25. Oct. 2021], https://docs.safegraph.com/docs/monthly-patterns.

[51] SafeGraph. Places API Overview, Nov 2021. [Online; accessed 11. Nov. 2021], https://docs.safegraph.com/reference\#places-api-overview-new.

[52] SafeGraph. Privacy Policy, Jul 2021. [Online; accessed 25. Oct. 2021], https://www.safegraph.com/privacy-policy.

[53] SPARber, S. Harris County mask order by Judge Lina Hidalgo faces pushback. Texas Tribune (Apr 2020). https://www.texastribune.org/2020/04/22/harris-county-maskorder-coronavirus. 
[54] Sparks, K., Thakur, G., Pasarkar, A., And Urban, M. A global analysis of cities geosocial temporal signatures for points of interest hours of operation. International Journal of Geographical Information Science 34, 4 (2020), 759-776. doi:10.1080/13658816.2019.1615069.

[55] TANG, J., SONG, Y., Miller, H. J., AND Zhou, X. Estimating the most likely spacetime paths, dwell times and path uncertainties from vehicle trajectory data: A time geographic method. Transportation Research Part C: Emerging Technologies 66 (2016), 176-194. doi:10.1016/j.trc.2015.08.014.

[56] Thomas, I., Frankhauser, P., AND Badariotti, D. Comparing the fractality of european urban neighbourhoods: do national contexts matter? Journal of Geographical Systems 14, 2 (2012), 189-208. doi:10.1007/s10109-010-0142-4.

[57] Tu, W., ZhU, T., XiA, J., ZhOU, Y., LAI, Y., JiAnG, J., AND LI, Q. Portraying the spatial dynamics of urban vibrancy using multisource urban big data. Computers, Environment and Urban Systems 80 (2020), 101428. doi:10.1016/j.compenvurbsys.2019.101428.

[58] Uitenbroek, D. G., Kerekovska, A., and Festchieva, N. Health lifestyle behaviour and socio-demographic characteristics. a study of varna, glasgow and edinburgh. Social science \& medicine 43, 3 (1996), 367-377. doi:10.1016/0277-9536(95)003991.

[59] United States Census Bureau. TIGER/Line Shapefile, 2017, 2010 nation, U.S., 2010 Census Urban Area National , 2017. https:/ / catalog.data.gov/dataset/tiger-lineshapefile-2017-2010-nation-u-s-2010-census-urban-area-national.

[60] United States Census Bureau. U.S. Median Household Income Was $\$ 63,179$ in 2018, Not Significantly Different From 2017. United States Census Bureau (Sep 2019).

[61] United States Census Bureau. American Community Survey, 2019American Community Survey 5-Year Estimates, Table DP03, Jul 2021. [Online; accessed 27. Jul. 2021].

[62] United States Census Bureau. American Community Survey, 2019American Community Survey 5-Year Estimates, Table DP05, Jul 2021. [Online; accessed 27. Jul. 2021].

[63] Verma, R., YABE, T., AND UKKUSURI, S. V. Spatiotemporal contact density explains the disparity of covid-19 spread in urban neighborhoods. Scientific Reports 11, 1 (2021), 1-11. doi:10.1038/s41598-021-90483-1.

[64] WarRen, M. S., AND SKILlman, S. W. Mobility changes in response to COVID-19. arXiv preprint arXiv:2003.14228 (2020).

[65] Yan, B., JanOwicz, K., MAI, G., AND GAO, S. From itdl to place2vec: Reasoning about place type similarity and relatedness by learning embeddings from augmented spatial contexts. In Proceedings of the 25th ACM SIGSPATIAL international conference on advances in geographic information systems (2017), pp. 1-10. doi:10.1145/3139958.3140054.

www.josis.org 
[66] Ye, M., JANOWicZ, K., MÜlligann, C., AND LeE, W.-C. What you are is when you are: the temporal dimension of feature types in location-based social networks. In Proceedings of the 19th ACM SIGSPATIAL International Conference on Advances in Geographic Information Systems (2011), pp. 102-111. doi:10.1145/2093973.2093989.

[67] Zhang, F., YuAN, N. J., ZhenG, K., LIAN, D., XIE, X., AND RUI, Y. Exploiting dining preference for restaurant recommendation. In Proceedings of the 25th International Conference on World Wide Web (2016), pp. 725-735. doi:10.1145/2872427.2882995.

\section{Appendices}

\section{Regression Results}

\begin{tabular}{lllll}
\hline & $\begin{array}{l}\text { New York City } \\
(\mathrm{N}=7128)\end{array}$ & $\begin{array}{l}\text { Los Angeles } \\
(\mathrm{N}=11271)\end{array}$ & $\begin{array}{l}\text { Chicago } \\
(\mathrm{N}=3668)\end{array}$ & $\begin{array}{l}\text { Houston } \\
(\mathrm{N}=6436)\end{array}$ \\
\hline Constant & 1.599 & $17.452^{* * *}$ & $22.864^{* * *}$ & 1.61 \\
& $(-4.979)$ & $(-4.014)$ & $(7.683)$ & $(-4.028)$ \\
Percent Female & $0.125^{* *}$ & $-0.138^{* * *}$ & $-0.185^{* *}$ & 0.042 \\
& $(0.051)$ & $(0.046)$ & $(0.075)$ & $(0.047)$ \\
Percent White & -0.015 & -0.013 & -0.023 & -0.004 \\
& $(0.032)$ & $(0.019)$ & $(0.054)$ & $(0.020)$ \\
Percent Black & $-0.072^{* *}$ & $-0.101^{* * *}$ & $-0.097^{*}$ & -0.026 \\
& $(0.034)$ & $(0.027)$ & $(0.059)$ & $(0.024)$ \\
Percent Asian & -0.047 & 0.012 & 0.052 & 0.024 \\
Percent Hispanic & $(0.037)$ & $(0.026)$ & $(0.069)$ & $(0.033)$ \\
Perc. Pop. 16+ in Labor Force & $0.017^{* * *}$ & $-0.069^{* * *}$ & -0.085 & -0.007 \\
& $(0.024)$ & $(0.013)$ & $(0.032)$ & $(0.014)$ \\
Median Household Income & 0.076 & $0.052^{* *}$ & 0.051 & 0.024 \\
& $(0.029)$ & $(0.025)$ & $(0.041)$ & $(0.024)$ \\
Median Family Income & $1.31 \mathrm{E}-05$ & $-7.06 \mathrm{E}-06$ & $-1.84 \mathrm{E}-05$ & $3.19 \mathrm{E}-06$ \\
& $(9.49 E-06)$ & $(1.21 E-05)$ & $(1.92 E-05)$ & $(1.19 E-05)$ \\
Perc. Below Poverty Line & $6.19 \mathrm{E}-07$ & $3.44 \mathrm{E}-06$ & $1.24 \mathrm{E}-05$ & $5.53 \mathrm{E}-06$ \\
& $(4.63 E-06)$ & $(9.92 \mathrm{E}-06)$ & $(1.07 E-05)$ & $(9.42 E-06)$ \\
& $0.053^{*}$ & $0.095^{* * *}$ & 0.020 & 0.041 \\
Lagrange Multiplier & $(0.031)$ & $(0.027)$ & $(0.046)$ & $(0.024)$ \\
Nagelkerke pseudo R-squared & $11.902^{* * *}$ & $24.981^{* * *}$ & $27.552^{* * *}$ & $27.544^{* * *}$ \\
Log likelihood & 0.012 & 0.016 & 0.044 & 0.007 \\
Akaike Information Criterion & -30554.52 & -47050.2 & -15879.77 & -25626.93 \\
\hline
\end{tabular}

${ }^{*} \mathrm{p}<0.1,{ }^{* *} \mathrm{p}<0.05,{ }^{* * *} \mathrm{p}<0.01$. The value in parenthesis is the standard error of the variable

Table 7: Spatial error regression model results for change in visit duration to All POI split by city. 


\begin{tabular}{|c|c|c|c|c|}
\hline & $\begin{array}{l}\text { New York City } \\
(\mathrm{N}=2437)\end{array}$ & $\begin{array}{l}\text { Los Angeles } \\
(\mathrm{N}=3636)\end{array}$ & $\begin{array}{l}\text { Chicago } \\
(\mathrm{N}=1337)\end{array}$ & $\begin{array}{l}\text { Houston } \\
(\mathrm{N}=2068)\end{array}$ \\
\hline \multirow[t]{2}{*}{ Constant } & 9.492 & $23.145^{* * *}$ & $28.798^{* *}$ & -0.588 \\
\hline & $(7.847)$ & $\begin{array}{l}(6.680) \\
0\end{array}$ & $\begin{array}{l}(12.409) \\
-0179\end{array}$ & $\begin{array}{l}(4.985) \\
0118 * *\end{array}$ \\
\hline Percent Female & $(0.080)$ & $(0.076)$ & $(0.117)$ & $(0.055)$ \\
\hline \multirow[t]{2}{*}{ Percent White } & -0.034 & 0.004 & -0.118 & -0.017 \\
\hline & $(0.050)$ & $(0.032)$ & $(0.086)$ & $(0.025)$ \\
\hline \multirow[t]{2}{*}{ Percent Black } & $-0.177^{* * *}$ & $-0.263^{* * *}$ & $-0.284^{* * *}$ & $-0.070^{* *}$ \\
\hline & $(0.054)$ & $(0.044)$ & $(0.093)$ & $(0.029)$ \\
\hline \multirow[t]{2}{*}{ Percent Asian } & -0.086 & 0.023 & -0.015 & 0.023 \\
\hline & $(0.058)$ & $(0.042)$ & $(0.107)$ & $(0.041)$ \\
\hline \multirow[t]{2}{*}{ Percent Hispanic } & -0.035 & $-0.161^{* * *}$ & $-0.135^{* * *}$ & $-0.041^{* *}$ \\
\hline & $(0.038)$ & $(0.021)$ & $(0.050)$ & $(0.018)$ \\
\hline \multirow[t]{2}{*}{ Perc. Pop. 16+ in Labor Force } & $0.172^{* * *}$ & $0.101^{* *}$ & $0.217^{* * *}$ & $0.079^{* * *}$ \\
\hline & $(0.047)$ & $(0.042)$ & $(0.063)$ & $(0.029)$ \\
\hline \multirow[t]{2}{*}{ Median Household Income } & $-2.89 \mathrm{E}-05^{*}$ & $-3.58 \mathrm{E}-05^{*}$ & $-6.38 \mathrm{E}-05^{* *}$ & $1.50 \mathrm{E}-05$ \\
\hline & $(1.54 E-05)$ & $(2.09 E-05)$ & $(2.81 E-05)$ & $(1.42 E-05)$ \\
\hline \multirow[t]{2}{*}{ Median Family Income } & $-3.55 E-06$ & 7.57E-06 & $2.89 \mathrm{E}-05^{*}$ & $-1.32 \mathrm{E}-05$ \\
\hline & $(7.10 E-06)$ & $(1.72 E-05)$ & $(1.59 E-05)$ & $(1.11 E-05)$ \\
\hline \multirow[t]{2}{*}{ Perc. Below Poverty Line } & -0.029 & $0.077^{*}$ & 0.024 & $0.050^{*}$ \\
\hline & $(0.049)$ & $(0.045)$ & $(0.075)$ & $(0.029)$ \\
\hline Lagrange Multiplier & $7.894^{* * *}$ & $5.781^{* *}$ & $8.424^{* * *}$ & $10.715^{* * *}$ \\
\hline Nagelkerke pseudo R-squared & 0.051 & 0.083 & 0.150 & 0.028 \\
\hline Log likelihood & -10208.12 & -15375.17 & -5688.074 & -7464.587 \\
\hline Akaike Information Criterion & 20440 & 30774 & 11400 & 14953 \\
\hline
\end{tabular}

${ }^{*} \mathrm{p}<0.1,{ }^{* *} \mathrm{p}<0.05,{ }^{* * *} \mathrm{p}<0.01$. The value in parenthesis is the standard error of the variable

Table 8: Spatial error regression model results for change in visit duration to the Restaurant place type, split by city. 


\begin{tabular}{|c|c|c|c|c|}
\hline & $\begin{array}{l}\text { New York City } \\
(\mathrm{N}=788)\end{array}$ & $\begin{array}{l}\text { Los Angeles } \\
(\mathrm{N}=813)\end{array}$ & $\begin{array}{l}\text { Chicago } \\
(\mathrm{N}=354)\end{array}$ & $\begin{array}{l}\text { Houston } \\
(\mathrm{N}=445)\end{array}$ \\
\hline Constant & $\begin{array}{l}-12.359 \\
(12.524)\end{array}$ & $\begin{array}{l}12.790 \\
(11.426)\end{array}$ & $\begin{array}{l}23.964 \\
(18.713)\end{array}$ & $\begin{array}{l}14.210 \\
(10.209)\end{array}$ \\
\hline Percent Female & $\begin{array}{l}0.133 \\
(0.121)\end{array}$ & $\begin{array}{l}-0.111 \\
(0.126)\end{array}$ & $\begin{array}{l}-0.171 \\
(0.165)\end{array}$ & $\begin{array}{l}-0.025 \\
(0.112)\end{array}$ \\
\hline Percent White & $\begin{array}{l}-0.003 \\
(0.082)\end{array}$ & $\begin{array}{l}-0.012 \\
(0.058)\end{array}$ & $\begin{array}{l}-0.064 \\
(0.145)\end{array}$ & $\begin{array}{l}-0.117^{* *} \\
(0.055)\end{array}$ \\
\hline Percent Black & $\begin{array}{l}-0.106 \\
(0.090)\end{array}$ & $\begin{array}{l}-0.100 \\
(0.077)\end{array}$ & $\begin{array}{l}-0.155 \\
(0.154)\end{array}$ & $\begin{array}{l}-0.176^{* * *} \\
(0.064)\end{array}$ \\
\hline Percent Asian & $\begin{array}{l}-0.020 \\
(0.096)\end{array}$ & $\begin{array}{l}0.003 \\
(0.072)\end{array}$ & $\begin{array}{l}0.000 \\
(0.172)\end{array}$ & $\begin{array}{l}-0.122 \\
(0.084)\end{array}$ \\
\hline Percent Hispanic & $\begin{array}{l}0.001 \\
(0.063)\end{array}$ & $\begin{array}{l}-0.158^{* * *} \\
(0.036)\end{array}$ & $\begin{array}{l}-0.180^{* *} \\
(0.080)\end{array}$ & $\begin{array}{l}-0.076^{* *} \\
(0.035)\end{array}$ \\
\hline Perc. Pop. $16+$ in Labor Force & $\begin{array}{l}0.265^{* * *} \\
(0.067)\end{array}$ & $\begin{array}{l}0.181^{* * *} \\
(0.064)\end{array}$ & $\begin{array}{l}0.110 \\
(0.090)\end{array}$ & $\begin{array}{l}0.068 \\
(0.057)\end{array}$ \\
\hline Median Household Income & $\begin{array}{l}4.57 \mathrm{E}-05^{* *} \\
(2.06 E-05)\end{array}$ & $\begin{array}{l}3.94 \mathrm{E}-06 \\
(2.61 E-05)\end{array}$ & $\begin{array}{l}-3.38 E-05 \\
(3.62 E-05)\end{array}$ & $\begin{array}{l}-4.22 \mathrm{E}-05^{*} \\
(2.57 E-05)\end{array}$ \\
\hline Median Family Income & $\begin{array}{l}-7.29 \mathrm{E}-06 \\
(9.77 E-06)\end{array}$ & $\begin{array}{l}-2.17 \mathrm{E}-05 \\
(2.05 E-05)\end{array}$ & $\begin{array}{l}2.59 \mathrm{E}-05 \\
(1.82 \mathrm{E}-05)\end{array}$ & $\begin{array}{l}4.09 \mathrm{E}-05^{*} \\
(2.11 E-05)\end{array}$ \\
\hline Perc. Below Poverty Line & $\begin{array}{l}0.13181^{*} \\
(0.078)\end{array}$ & $\begin{array}{l}0.094 \\
(0.071)\end{array}$ & $\begin{array}{l}-0.027 \\
(0.106)\end{array}$ & $\begin{array}{l}0.099 * \\
(0.060)\end{array}$ \\
\hline Lagrange Multiplier & 0.495 & $6.236^{* *}$ & $6.794^{* * *}$ & 0.208 \\
\hline Nagelkerke pseudo R-squared & 0.085 & 0.107 & 0.176 & 0.060 \\
\hline Log likelihood & -3212.275 & -3195.535 & -1407.649 & -1571.515 \\
\hline Akaike Information Criterion & 6448.5 & 6415.1 & 2839.3 & 3167 \\
\hline
\end{tabular}

${ }^{*} \mathrm{p}<0.1,{ }^{* *} \mathrm{p}<0.05,{ }^{* * *} \mathrm{p}<0.01$. The value in parenthesis is the standard error of the variable

Table 9: Spatial error regression model results for change in visit duration to the Coffee Shop, place type split by city. 


\begin{tabular}{lllll}
\hline & $\begin{array}{l}\text { New York City } \\
(\mathrm{N}=135)\end{array}$ & $\begin{array}{l}\text { Los Angeles } \\
(\mathrm{N}=610)\end{array}$ & $\begin{array}{l}\text { Chicago } \\
(\mathrm{N}=130)\end{array}$ & $\begin{array}{l}\text { Houston } \\
(\mathrm{N}=468)\end{array}$ \\
\hline Constant & 0.696 & -0.841 & 0.519 & -1.286 \\
& $(2.938)$ & $(2.951)$ & $(5.233)$ & $(3.586)$ \\
Percent Female & 0.003 & -0.002 & -0.107 & 0.032 \\
& $(0.031)$ & $(0.033)$ & $(0.063)$ & $(0.045)$ \\
Percent White & -0.004 & 0.005 & $0.057^{*}$ & 0.007 \\
Percent Black & $(0.017)$ & $(0.012)$ & $(0.032)$ & $(0.020)$ \\
& -0.012 & 0.004 & $0.061^{*}$ & 0.035 \\
Percent Asian & $(0.020)$ & $(0.016)$ & $(0.035)$ & $(0.023)$ \\
& -0.008 & 0.001 & 0.055 & -0.003 \\
Percent Hispanic & $(0.022)$ & $(0.016)$ & $(0.045)$ & $(0.032)$ \\
Perc. Pop. 16+ in Labor Force & -0.012 & -0.002 & $0.034^{*}$ & 0.011 \\
& $(0.014)$ & 0.008 & $(0.020)$ & $(0.013)$ \\
Median Household Income & 0.009 & 0.008 & 0.011 & -0.028 \\
& $(0.020)$ & $(0.017)$ & $(0.029)$ & $(0.022)$ \\
Median Family Income & $5.49 \mathrm{E}-06$ & $6.58 \mathrm{E}-06$ & $-1.62 \mathrm{E}-05$ & $-1.24 \mathrm{E}-05$ \\
& $(1.43 \mathrm{E}-05)$ & $(9.37 \mathrm{E}-06)$ & $(2.22 \mathrm{E}-05)$ & $(1.29 E-05)$ \\
Perc. Below Poverty Line & $-7.35 \mathrm{E}-06$ & $-5.34 \mathrm{E}-06$ & $7.62 \mathrm{E}-07$ & $1.58 \mathrm{E}-05$ \\
& $(1.18 \mathrm{E}-05)$ & $(7.99 \mathrm{E}-06)$ & $(1.46 E-05)$ & $(1.03 E-05)$ \\
& $-3.17 \mathrm{E}-04$ & 0.028 & -0.024 & 0.005 \\
Lagrange Multiplier & $(0.019)$ & $(0.018)$ & $(0.026)$ & $(0.022)$ \\
Nagelkerke pseudo R-squared & 0.276 & 0.007 & 0.772 & 1.383 \\
Log likelihood & 0.021 & 0.007 & 0.077 & 0.031 \\
Akaike Information Criterion & -248.112 & -1459.275 & -297.605 & -1202.231 \\
\hline
\end{tabular}

${ }^{*} \mathrm{p}<0.1,{ }^{* *} \mathrm{p}<0.05,{ }^{* * *} \mathrm{p}<0.01$. The value in parenthesis is the standard error of the variable

Table 10: Spatial error regression model results for change in visit duration to the Fuel Station place type, split by city. 